\title{
Prospective Interventional Cohort Study of Ocular Surface Disease Changes in Eyes After Trabecular Micro-Bypass Stent(s) Implantation (iStent or iStent inject) with Phacoemulsification
}

\author{
Justin A. Schweitzer (D) - Whitney H. Hauser • Mitch Ibach • \\ Brandon Baartman - Subba R. Gollamudi - Andrew W. Crothers • \\ John E. Linn · John P. Berdahl
}

Received: July 5, 2020 / Published online: August 13, 2020

(C) The Author(s) 2020

\section{ABSTRACT}

Introduction: This study sought to assess ocular surface disease changes following cataract surgery combined with trabecular micro-bypass stent(s) implantation (iStent or iStent inject).

Methods: This prospective interventional single-arm clinical trial enrolled 47 eyes with mildto-moderate open-angle glaucoma (OAG) on 1-4 glaucoma medications who underwent phacoemulsification and trabecular micro-bypass stent(s) implantation. Key glaucoma and ocular surface data through 3 months postoperatively included the Ocular Surface Disease Index score (OSDI), corneal/conjunctival staining (Oxford Schema), fluorescein tear break-up time (FTBUT), conjunctival hyperemia (Efron Scale), glaucoma medications, and intraocular pressure (IOP).

Digital Features To view digital features for this article go to https://doi.org/10.6084/m9.figshare.12753305.

J. A. Schweitzer $(\varangle) \cdot$ M. Ibach · B. Baartman .

J. P. Berdahl

Vance Thompson Vision, Sioux Falls, SD, USA e-mail:

justin.schweitzer@vancethompsonvision.com

W. H. Hauser - S. R. Gollamudi · A. W. Crothers ·

J. E. Linn

Eye Specialty Group, Memphis, TN, USA
Results: Mean OSDI scores improved from $40.1 \pm 21.6 \quad$ (severe) preoperatively to $17.5 \pm 15.3$ (mild) at 3 months $(p<0.0001)$. While $73 \%$ of eyes had moderate or severe OSDI scores preoperatively, $29 \%$ had such scores at 3 months, and the OSDI score was normal in $57 \%$ of eyes versus $9 \%$ preoperatively. Mean FTBUT increased from $4.3 \pm 2.4 \mathrm{~s}$ preoperatively to $6.4 \pm 2.5 \mathrm{~s}$ at 3 months $(p<0.0001)$; mean Oxford corneal/conjunctival staining reduced from $1.4 \pm 1.0$ preoperatively to $0.4 \pm 0.6$ at 3 months $(p<0.0001)$; mean Efron conjunctival hyperemia score reduced from $1.4 \pm 0.7$ preoperatively to $1.2 \pm 0.6$ at 3 months $(p=0.118)$. The number of glaucoma medications decreased from $1.5 \pm 0.9$ to $0.6 \pm 0.8$ mean medications $(60 \%$ reduction, $p<0.0001)$, with all eyes maintaining or reducing medications versus preoperatively and $55 \%$ of eyes becoming medication-free (versus $0 \%$ preoperatively). Mean IOP reduced from $17.4 \pm 4.2 \mathrm{mmHg}$ to $14.5 \pm 3.2 \mathrm{mmHg}$ $(p<0.0001)$. The safety profile was excellent.

Conclusion: Implantation of trabecular microbypass stent(s) (iStent or iStent inject) with cataract surgery produced significant improvements in ocular surface health, alongside significant reductions in IOP and medications.

Trial registration: ClinicalTrials.gov identifier, NCT04452279.

Keywords: Dry eye; Medication; MIGS; Ocular surface; Quality of life; Trabecular micro-bypass 


\section{Key Summary Points}

\section{Why carry out this study?}

Topical glaucoma medications are widely known to cause or worsen ocular surface disease, while trabecular micro-bypass stent implantation with iStent or iStent inject is proven to consistently reduce medication burden and intraocular pressure (IOP) in glaucomatous eyes.

This prospective cohort study is one of the first to examine the direct linkage between stent implantation with

phacoemulsification and postoperative changes in ocular surface disease.

Specifically, this 3-month study measured the Ocular Surface Disease Index score (OSDI), corneal/conjunctival staining (Oxford Schema), fluorescein tear breakup time (FTBUT), conjunctival hyperemia (Efron Scale), glaucoma medications, and IOP.

\section{What was learned from the study?}

Implantation of iStent or iStent inject with cataract surgery produced meaningful improvements in all measures of ocular surface health, alongside significant reductions in IOP and medications.

Specifically, mean OSDI score improved from 40.1 (severe) to 17.5 (mild) ( $p<$ 0.0001 ), with a $\sim 2.5$-fold decrease in the proportion of eyes with moderate/severe OSDI and a $\sim 6$-fold increase in the proportion of eyes with normal OSDI. Mean FTBUT increased from 4.3 to $6.4 \mathrm{~s}$ $(p<0.0001)$; mean Oxford corneal/conjunctival staining reduced from 1.4 to 0.4 ( $p<0.0001)$; mean Efron conjunctival hyperemia reduced from 1.4 to $1.2(p=0.118)$.

Meanwhile, the mean number of glaucoma medications decreased from 1.5 to 0.6 medications $(p<0.0001)$; mean IOP reduced from 17.4 to $14.5 \mathrm{mmHg}$ ( $p<$ $0.0001)$; the safety profile was excellent.

\section{INTRODUCTION}

Ocular surface disease (OSD), a top cause of patient visits to eye care providers, is characterized by inadequate tear quantity, unstable tear film, and ocular surface breakdown as well as symptoms of dryness, burning, photophobia, irritation, and blurred vision $[1,2]$. The prevalence of OSD within the USA is estimated to be approximately $15 \%$ in individuals aged $\geq$ 65 years [3]. However, OSD is more frequent in patients with glaucoma, with reported rates of disease in up to 59\% [4] of patients and OSD symptoms in up to $74 \%$ of patients [5]. A major reason cited for this higher prevalence is the use of preservative-containing eyedrops, which are known to promote the development or worsening of OSD [6-9]. Ocular surface disease, in turn, has been shown to reduce patient adherence to therapy $[10,11]$, increase the risk of future glaucoma surgical failure $[12,13]$, and worsen patient quality of life $[1,14,15]$.

As an alternative to topical medications for glaucoma treatment, patients may undergo a range of interventions, from more conservative laser procedures on one end of the spectrum to more invasive filtering surgeries (e.g., trabeculectomy, tube shunt) on the other. Between these two extremes, micro-invasive glaucoma surgery (MIGS) has gained a growing role in the glaucoma treatment algorithm. The first US FDA-approved MIGS technology, the iStent trabecular micro-bypass (Glaukos Corp., San Clemente, CA), has the most published evidence of any MIGS device in patients with glaucoma. The more recent second-generation iStent inject, FDA-approved in 2018, is being increasingly utilized as well. Both micro-scale devices are designed to create a patent pathway through the diseased trabecular meshwork into Schlemm's canal, thereby increasing aqueous humor outflow from the anterior chamber and decreasing intraocular pressure (IOP). Stent implantation has been examined in the setting of cataract surgery or as a standalone procedure, in varying glaucoma severities ranging from glaucoma suspect to refractory glaucoma, and in various glaucoma subtypes (e.g., pseudoexfoliative, pigmentary, normal-tension) as well as 
in primary open-angle glaucoma (POAG) [16-31]. In both long- and short-term followup, stent implantation has consistently demonstrated substantial reductions in IOP and medication burden. Despite this substantive evidence base showing medication reduction after iStent or iStent inject implantation, to our knowledge, no publication to date has exclusively examined the effect of stent implantation on OSD after surgery.

The current prospective study evaluates OSD parameters through 3 months following iStent or iStent inject implantation in combination with cataract surgery in patients with mild-tomoderate OAG treated with topical medication(s). The study uses four of the most widely accepted and well-validated measures of ocular surface health: the Ocular Surface Disease Index (OSDI) patient questionnaire [32], Oxford Schema for corneal/conjunctival staining [33], Efron Scale for conjunctival hyperemia [34], and fluorescein tear break-up time (FTBUT). The OSDI is broadly referenced in the literature $[1-5,8,9,32]$ and is the most widely used test in OSD clinical trials; it is regarded as the gold standard OSD diagnostic test because of its high reliability and validity [32], and it is the consensus recommendation of the Tear Film and Ocular Surface Society Dry Eye Workshop II (TFOS DEWS II) Diagnostic Methodology Subcommittee due to the test's strong establishment in the field [35]. The present study used these tests to prospectively examine the connection between stent implantation with phacoemulsification and postoperative changes in ocular surface disease.

\section{METHODS}

\section{Study Design, Participants, and Data Analysis}

This prospective interventional multi-surgeon study enrolled participants at two private ophthalmology centers in the US. Consecutive patients scheduled for stent-cataract surgery were asked if they were interested in participating in the study, in which case they completed the OSDI questionnaire to determine study eligibility. Inclusion criteria consisted of a diagnosis of mild-to-moderate open-angle glaucoma (including primary open-angle, pseudoexfoliative, and pigmentary), cataract requiring surgery, current treatment with one or more ocular hypotensive medications, and baseline OSDI score $\geq 8$.

Ethics approval was obtained from the Institutional Review Board (IRB) of the Southern College of Optometry (Memphis, TN, USA). All procedures performed were in accordance with the 1964 Declaration of Helsinki and its later amendments or comparable ethical standards. Informed consent was obtained from all participants. The study was registered in the clinicaltrials.gov registry (\#NCT04452279).

The principal OSD efficacy measures were scores on the OSDI questionnaire, Oxford Schema for corneal/conjunctival staining, Efron Scale for conjunctival hyperemia, and FTBUT. Standard glaucoma outcomes of IOP and medications also were recorded. Patients were followed for 3 months postoperatively, with efficacy outcomes compared at 3 months vs. preoperatively. Pre- and postoperative OSD measurements were compared using paired t-tests, with the significance threshold set at a $p$ value $<0.05$. Continuous variables are presented as mean \pm standard deviation, and categorical variables are measured in terms of proportions.

\section{OSD Testing Protocols}

The four OSD tests and their respective grading scales are depicted in Fig. 1a-d. The OSDI is a patient-completed questionnaire, while the other three tests are determined by the clinician at the slit lamp. The OSDI (Fig. 1a) is a 12-item subjective questionnaire designed to assess a patient's symptoms related to chronic dry eye, its severity, and its impact on ability to function [32]. OSDI scores range from 0 to 100 , with severity defined as normal (0-12), mild (13-22), moderate (23-32), and severe (33-100). The Oxford Schema (Fig. 1b) is based on the level of lissamine green and sodium fluorescein staining of the cornea and conjunctiva, as observed after instillation of each solution to the non- 
a

Ocular Surface Disease Index (OSDI)

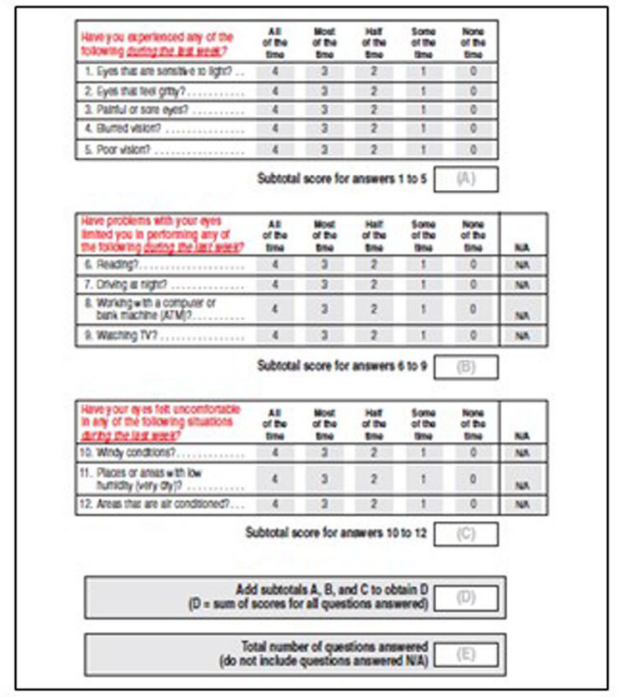

C Efron Scale for Conjunctival Hyperemia

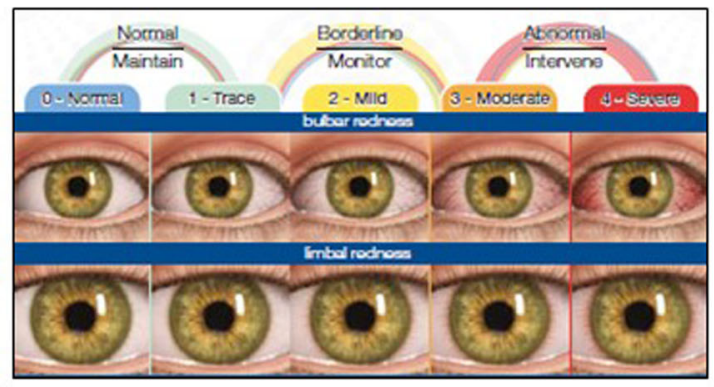

Fig. 1 a Ocular Surface Disease Index (OSDI). b Oxford schema for corneal and conjunctival staining. c Efron Scale for conjunctival hyperemia. d Sample images of fluorescein

anesthetized lower palpebral conjunctiva [33]. The Efron Scale (Fig. 1c) is used to classify conjunctival hyperemia according to standardized images; it is scored from 0 (normal) to 4 (severe) [34]. The FTBUT (Fig. 1d) is tested by instilling approximately $5 \mu \mathrm{l}$ of $2 \%$ sodium fluorescein solution into the inferior cul-de-sac, asking the patient to blink normally, and measuring the time for tear break-up under non-anesthetized conditions. FTBUT is classified as normal ( $\geq 10 \mathrm{~s}$ ), mild to moderate (5-9s), or severe $(<5 s)[4]$. b

Oxford Schema for Corneal and Conjunctival Staining

\begin{tabular}{|c|c|c|c|c|c|}
\hline \multicolumn{6}{|c|}{ 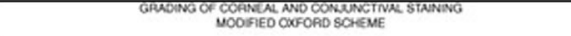 } \\
\hline PANEL & CPNOE & CAITERIA & $\begin{array}{l}\text { Dot couNT } \\
\text { (per woctor) }\end{array}$ & 100 & $\begin{array}{l}\text { VEREN } \\
\text { DESCFUPTIOA }\end{array}$ \\
\hline & 0 & $\begin{array}{l}\text { Eowal to o loss } \\
\text { inim desed A }\end{array}$ & 1 & 0 & Noseren \\
\hline & 1 & 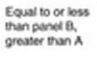 & 10 & 10 & Momal \\
\hline & " & $\begin{array}{l}\text { Eowal to of loss } \\
\text { then posed C. } \\
\text { gromer then B }\end{array}$ & 32 & is & mos \\
\hline & u & 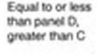 & 100 & 20 & Moserate \\
\hline & n & 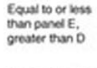 & 316 & 2.5 & Moneds \\
\hline$x$ & $\mathrm{v}$ & 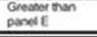 & 316 & 225 & Sevore \\
\hline
\end{tabular}

d Sample Images of Fluorescein Tear Break-Up Time (FTBUT) Assessment ${ }^{\mathrm{a}}$

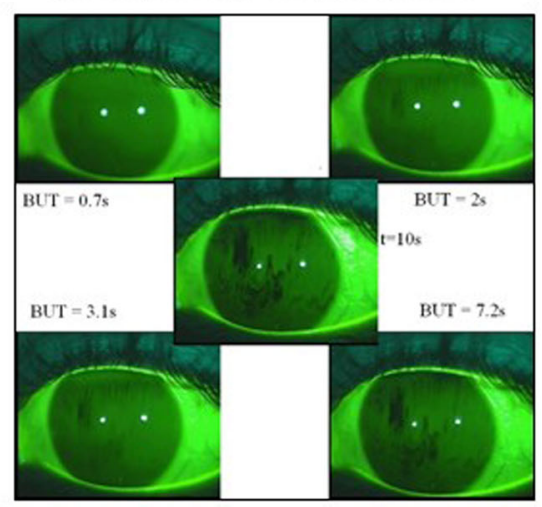

a FTBUT classifications: Normal, $\geq 10$ seconds; Mild to Moderate, 5 to 9 seconds; Severe, $<5$ seconds

tear break-up time (FTBUT) assessment ${ }^{\mathrm{a}}$. Footnote: ${ }^{a}$ FTBUT classifications: normal, $\geq 10 \mathrm{~s}$; mild to moderate, 5-9 s; severe, $<5 \mathrm{~s}$

\section{iStent and iStent Inject Devices, Implantation Technique, and Postoperative Medication Regimen}

The iStent and iStent inject are biocompatible, titanium, micro-scale stents designed to provide a patent bypass through the trabecular meshwork into Schlemm's canal, thereby facilitating aqueous outflow and decreasing IOP. The dimensions and design of each device have been detailed previously [16, 24]. Following successful cataract surgery, the surgeon advanced $a b$ internally through the existing 
phacoemulsification incision to the nasal trabecular meshwork, where he/she implanted either the iStent device (1 first-generation stent) or the iStent inject ( 2 s-generation stents). At the close of surgery, some eyes (40/47) received an intracameral injection of DexMoxiKetor (Imprimis Pharma, San Diego, CA), followed by once-daily topical PredGatiBrom or PredGatiNep (Imprimis Pharma for both) for 1 month. Eyes that did not receive an intraoperative injection were prescribed a topical regimen of antibiotic for 1 week, NSAID for 1 month, and corticosteroid taper over 1 month.

\section{RESULTS}

\section{Study Participants}

All eyes $(n=47)$ underwent uncomplicated phacoemulsification cataract surgery with concomitant iStent $(n=45)$ or iStent inject $(n=2)$ implantation. Mean baseline age was 70.9 years, approximately $57 \%$ of eyes were from female patients, and all patients were using topical glaucoma medication (range 1-4 medications). Mean preoperative OSD grades ranged from severe (OSDI 40.1) to mild (Oxford 1.4 and Efron 1.4), and mean FTBUT (4.3 s) was less than half the normal value of $\geq 10$ s. Preoperative demographic and clinical characteristics are shown in Table 1.

\section{Efficacy}

Significant improvements in OSDI, corneal/conjunctival staining, and fluorescein tear break-up time were observed at 3 months compared to preoperatively. The mean preoperative OSDI score was $40.1 \pm 21.6$, which decreased to $17.5 \pm 15.3$ at 3 months postoperatively ( $p<0.0001)$ (Fig. $2 \mathrm{a})$; this equated to reducing the mean OSDI severity from severe to mild disease. While $73 \%$ of eyes had moderate or severe OSDI scores preoperatively, only $29 \%$ had such scores at 3 months; by 3 months, 57\% of eyes had an OSDI score in the normal range (vs. $9 \%$ preoperatively) (Fig. 2b). Mean
Table 1 Baseline demographic and ocular characteristics

\begin{tabular}{ll}
\hline Baseline characteristics & $\boldsymbol{n}=\mathbf{4 7}$ \\
\hline Age (years; mean $\pm \mathrm{SD})$ & $70.9 \pm 6.8$ \\
Gender $(\% \mathrm{M} / \mathrm{F})$ & $43 \% / 57 \%$ \\
Race & $94 \%$ White $(44 /$ \\
& $47)$ \\
& $6 \%$ African \\
& American $(3 / 47)$
\end{tabular}

Surgery

iStent + phaco

$45(96 \%)$

iStent inject + phaco

$2(4 \%)$

IOP $(\mathrm{mmHg})$

$17.4 \pm 4.2$

Mean \pm SD

Glaucoma medications

Mean \pm SD

$1.5 \pm 0.9$

Range

Prescription ocular surface

$2.1 \%(1 / 47)^{\mathrm{a}}$

medications \% (n)

Ocular Surface Disease Index

(OSDI)

Mean \pm SD

$40.1 \pm 21.6$

Range

OSDI quartiles

0-12 Normal

$4(9 \%)$

13-22 Mild

$9(19 \%)$

23-32 Moderate

$6(13 \%)$

33-100 Severe

$28(60 \%)$

Conjunctival hyperemia (Efron

Scale)

Mean \pm SD

$1.4 \pm 0.7$

Range

$(0-3)$

Efron severities

0: Normal, no action required $3(6 \%)$

1: Trace; action rarely required $22(47 \%)$

2: Mild; action may be required $21(45 \%)$ 
Table 1 continued

\begin{tabular}{|c|c|}
\hline Baseline characteristics & $n=47$ \\
\hline $\begin{array}{l}\text { 3: Moderate; action usually } \\
\text { required }\end{array}$ & $1(2 \%)$ \\
\hline 4: Severe; action certainly required & $0(0 \%)$ \\
\hline \multicolumn{2}{|l|}{$\begin{array}{l}\text { Corneal/conjunctival staining } \\
\text { (Oxford Schema) }\end{array}$} \\
\hline Mean \pm SD & $1.4 \pm 1.0$ \\
\hline Range & $(0-3)$ \\
\hline \multicolumn{2}{|l|}{ Oxford severities } \\
\hline 0: Absent & $9(19 \%)$ \\
\hline 1: Minimal & $19(40 \%)$ \\
\hline 2: Mild & $12(26 \%)$ \\
\hline 3: Moderate & $7(15 \%)$ \\
\hline 4: Marked & $0(0 \%)$ \\
\hline 5: Severe & $0(0 \%)$ \\
\hline \multicolumn{2}{|l|}{$\begin{array}{l}\text { Fluorescein tear break-up time } \\
\qquad(\text { FTBUT) in (seconds) })^{\mathrm{b}}\end{array}$} \\
\hline Mean $\pm S D$ & $4.3 \pm 2.4$ \\
\hline
\end{tabular}

preoperative FTBUT was $4.3 \pm 2.4 \mathrm{~s}$, less than half of the normal value of $\geq 10 \mathrm{~s}$. At 3 months, mean FTBUT had increased to $6.4 \pm 2.5 \mathrm{~s}$ $(p<0.0001) \quad$ (Fig. 3a). The mean Oxford corneal/conjunctival staining score also reduced significantly, from $1.4 \pm 1.0$ preoperatively to $0.4 \pm 0.6$ at 3 months $(p<0.0001)$ (Fig. 3b). The mean Efron score for conjunctival hyperemia reduced from $1.4 \pm 0.7$ preoperatively to $1.2 \pm 0.6$ at 3 months $(p=0.118)$ (Fig. 3c).

Medication burden pre- and postoperatively is shown in Fig. 4a-c. At 3 months postoperatively, mean medication burden decreased from $1.5 \pm 0.9$ to $0.6 \pm 0.8$ medications $(60 \%$ reduction, $p<0.0001)$. All eyes either maintained or decreased their medication regimen versus preoperatively, with $55 \%$ of eyes eliminating medications entirely (versus 0\% medication-free preoperatively). By 3 months, $72 \%$ of eyes had eliminated 1-3 medications from their preoperative regimen, and only $12 \%$ of eyes were on $\geq 2$ medications (versus 30\% preoperatively). Meanwhile, mean IOP reduced significantly, from $17.4 \pm 4.2 \mathrm{mmHg}$ to $14.5 \pm 3.2 \mathrm{mmHg}$ (17\% reduction, $p<0.0001)$ (Fig. 5).

Ocular surface measurements, IOP, and medications also were analyzed for the subgroup of eyes undergoing iStent implantation with phacoemulsification (i.e., excluding two iStent inject cases). Outcomes were similar to those of the overall cohort; see Table 2.

\section{Safety}

Safety parameters were highly favorable. All eyes were successfully implanted with iStent or iStent inject with concomitant cataract surgery; there were no intraoperative complications. Through 3 months postoperatively, no adverse events were reported. Notably, there were no reported cases of persistent inflammation, peripheral anterior synechiae (PAS), hypotony, choroidal hemorrhage, hyphema, or corneal decompensation. No eyes underwent additional glaucoma surgery during follow-up.

\section{DISCUSSION}

This prospective study in patients undergoing trabecular micro-bypass implantation (iStent or iStent inject) contributes some of the first data specifically analyzing the effect of a MIGS device on ocular surface health in patients with glaucoma. Three months after iStent or iStent inject implantation with cataract surgery, clinically and statistically significant improvements were observed in nearly all ocular surface parameters, including OSDI, corneal/conjunctival staining, and tear break-up time. The patient-level significance of these changes is that patients' average OSDI severity improved from severe to mild, mean corneal staining decreased more than threefold, and eyes on 
a

Mean Ocular Surface Disease Index (OSDI) Score Preoperative vs. Month 3

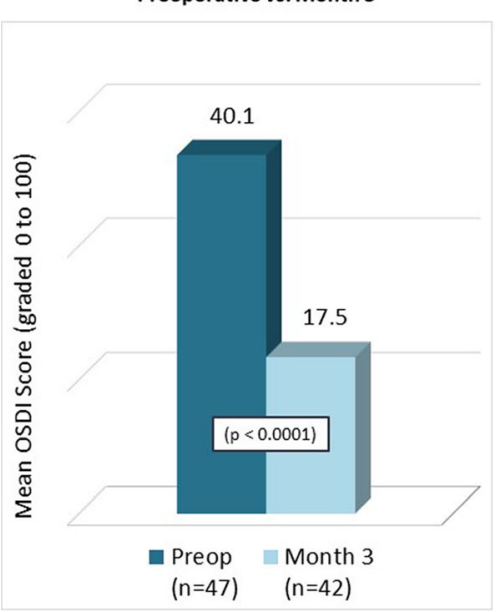

b

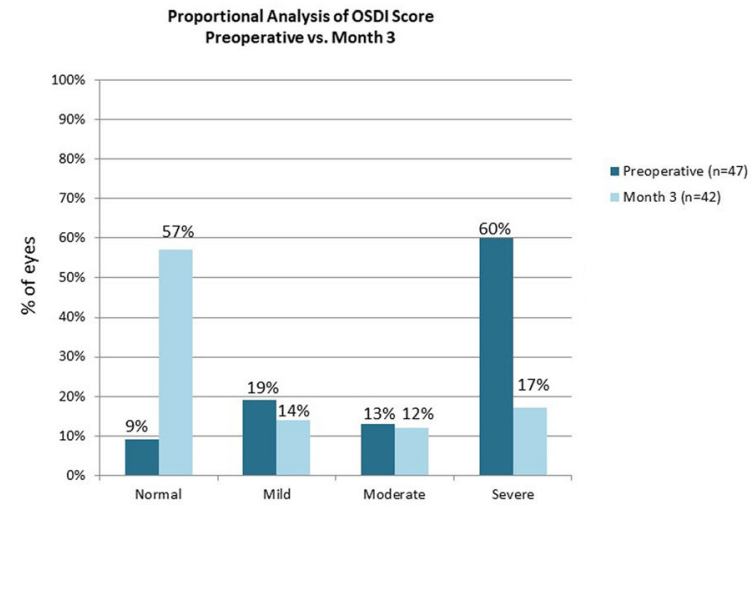

Fig. 2 a Mean Ocular Surface Disease Index (OSDI) score. Preoperative vs. month 3. b Proportional analysis of OSDI score. Preoperative vs. month 3
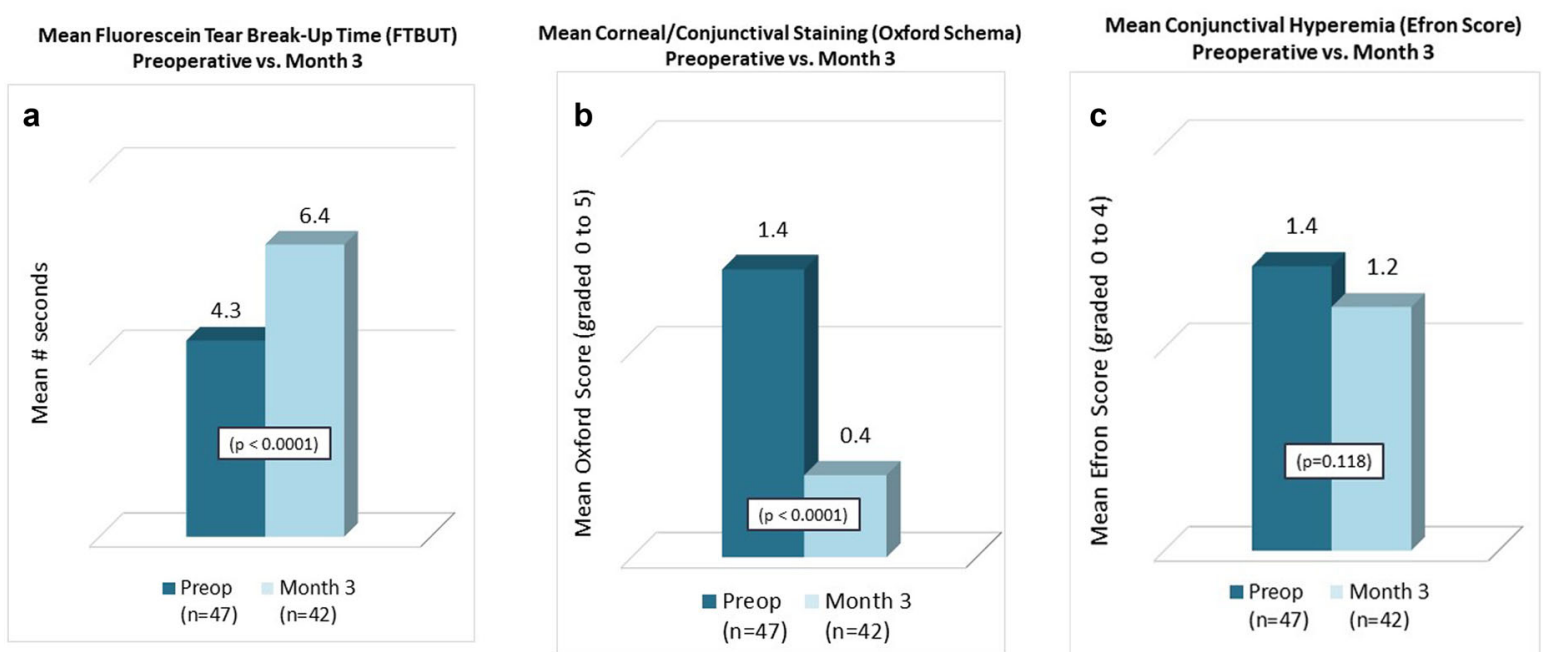

Fig. 3 a Mean fluorescein tear break-up time (FTBUT). Preoperative vs. month 3. b Mean corneal/conjunctival staining (Oxford Schema). Preoperative vs. month 3. c Mean conjunctival hyperemia (Efron score). Preoperative vs. month 3

average remained lubricated approximately $50 \%$ longer before break-up of tears, respectively. Conjunctival hyperemia also decreased, although this did not reach statistical significance (preoperative score was already in the Trace range).

In nearly all OSD studies, a central challenge is that patient symptoms (as measured by OSDI) do not necessarily match clinical signs (as measured by Oxford, Efron, FTBUT). However, the improvement of each measurement over time for a given patient is meaningful even if there is imperfect correlation between the various tests. By collecting such OSD data alongside traditional glaucoma outcomes (i.e., IOP, medications), this study may aid surgeons and patients in understanding the ocular surface effects of iStent and iStent inject implantation. 

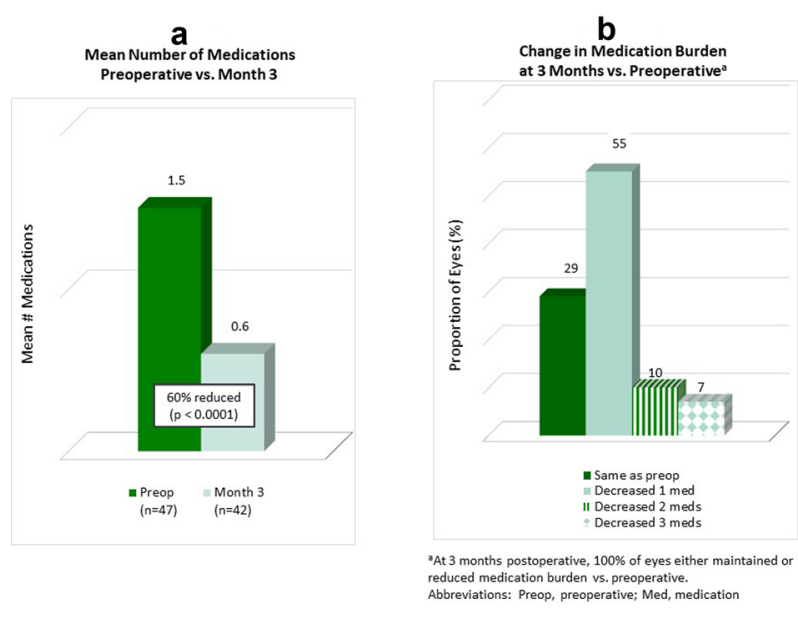

Fig. 4 a Mean number of medications. Preoperative vs. month 3. b Change in medication burden at 3 months vs. preoperatively ${ }^{a}$. Footnotes: ${ }^{a}$ At 3 months postoperatively, $100 \%$ of eyes either maintained or reduced medication

Mean Intraocular Pressure (IOP) Preoperative vs. Month 3

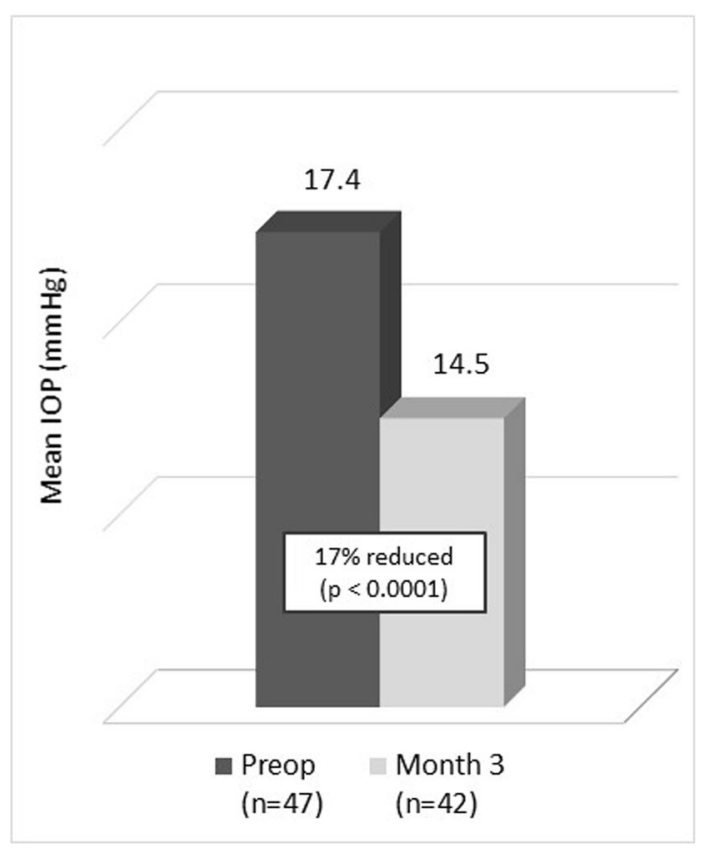

Fig. 5 Mean intraocular pressure (IOP). Preoperative vs. month 3

Any observed effects may be expected to apply specifically to iStent and iStent inject devices, given they are the smallest available trabecular

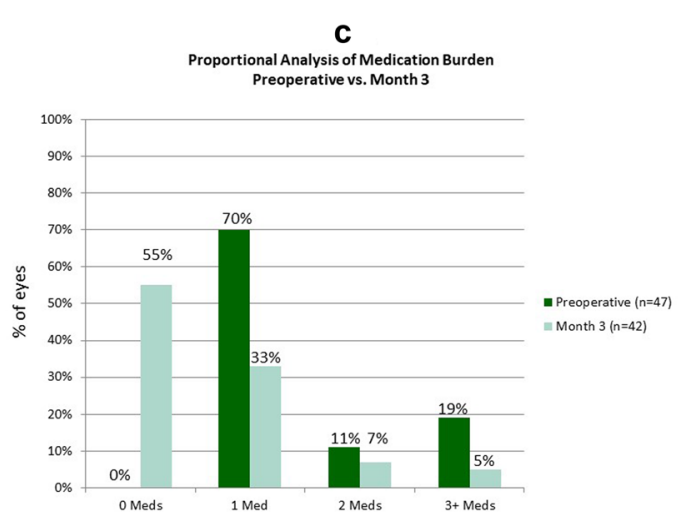

burden vs. preoperatively. Preop, preoperative; Med, medication. c Proportional analysis of medication burden. Preoperative vs. month 3

meshwork implants and have a longstanding evidence base showing favorable safety and minimal tissue disruption and inflammation. The same ocular surface benefits may not necessarily be transferrable to larger more invasive devices and procedures that could cause hyperemia and/or inflammatory cascades counteracting ocular surface improvements. Thus, any positive ocular surface effects may become part of the benefit-risk consideration of various medical and surgical treatment options for glaucoma.

Of the four OSD parameters measured in this study, the most dramatic improvements were observed in patients' OSDI scores. Given the preeminence of this test in OSD clinical studies $[32,35]$, and its widespread utilization in clinical practice, these improvements are especially promising. It also deserves mention that by 3 months postoperatively, significant improvements were observed in both subjective symptoms (e.g., OSDI) and clinical signs (e.g., FTBUT and Oxford staining). In other words, clinically detectable changes were apparent to the eye care provider, but patients also felt a tangible improvement in their symptoms (and conceivably in their quality of life). A similar correspondence between subjective and objective findings was demonstrated in a recent study by 
Table 2 Preoperative and month 3 ocular surface parameters, intraocular pressure, and number of medications eyes undergoing istent + phacoemulsification $(n=45)^{\mathrm{a}}$

\begin{tabular}{|c|c|c|c|c|c|c|}
\hline & $\begin{array}{l}\text { Conjunctival } \\
\text { hyperemia } \\
\text { (Efron Scale) }\end{array}$ & $\begin{array}{l}\text { Corneal/conjunc- } \\
\text { tival staining } \\
\text { (Oxford Schema) }\end{array}$ & $\begin{array}{l}\text { FTBUT (no. } \\
\text { seconds) }\end{array}$ & $\begin{array}{l}\text { Ocular } \\
\text { Surface } \\
\text { Disease Index } \\
\text { (OSDI) }\end{array}$ & $\begin{array}{l}\text { Intraocular } \\
\text { pressure } \\
(\mathbf{m m H g})\end{array}$ & $\begin{array}{l}\text { Number of } \\
\text { medications }\end{array}$ \\
\hline Preoperative & $1.4 \pm 0.7$ & $1.4 \pm 1.0$ & $4.4 \pm 2.4$ & $39.8 \pm 21.9$ & $17.5 \pm 4.2$ & $1.5 \pm 0.9$ \\
\hline Month 3 & $1.2 \pm 0.6$ & $0.4 \pm 0.5$ & $6.5 \pm 2.5$ & $16.4 \pm 14.8$ & $14.5 \pm 3.3$ & $0.6 \pm 0.8$ \\
\hline $\begin{array}{l}p \text { value vs. } \\
\text { preoperatively } \\
\text { (paired t-test) }\end{array}$ & 0.128 & $<0.0001$ & $<0.0001$ & $<0.0001$ & $<0.001$ & $<0.0001$ \\
\hline $\begin{array}{l}\text { \% Change vs. } \\
\text { preoperatively }\end{array}$ & $14 \%$ reduction & $71 \%$ reduction & $\begin{array}{l}\text { 48\% increase } \\
\text { (improvement) }\end{array}$ & $56 \%$ reduction & $\begin{array}{l}17 \% \\
\text { reduction }\end{array}$ & $\begin{array}{l}60 \% \\
\text { reduction }\end{array}$ \\
\hline
\end{tabular}

a Analysis does not include two eyes receiving iStent inject

Agnifili et al. [36], which showed OSDI scores to be directly correlated to the tear meniscus height and area measured by anterior segment optical coherence tomography (AS-OCT). The findings of both the present study and that of Agnifili et al. are notable given that in many OSD studies, symptoms do not necessarily correlate with objective measures [37].

Likely the primary factor contributing to these ocular surface improvements after iStent or iStent inject implantation is the significant reduction in medications, which themselves are known to promote or worsen OSD (6-9). The negative ocular surface effects of medications are widely documented. For example, Baudouin et al. demonstrated that longer duration of exposure to glaucoma medication(s) resulted in significant increases in ocular surface inflammatory markers such as immunoglobulin $\mathrm{E}$ (IgE) and class II human leukocyte antigen-D related antigen (HLA-DR) [6]. The same investigators also showed increased inflammatory markers with multiple topical glaucoma medications as compared to monotherapy [7]. Similarly, Rossi et al. demonstrated a clear relationship between dry eye disease and number of glaucoma medications, with dry eye present in $11 \%$ of eyes on one eyedrop compared to $39 \%$ and $43 \%$ of eyes on two and three drops, respectively [8].
Fechtner et al. also reported incrementally higher OSDI scores in patients using more glaucoma medications, with mean OSDI scores of $12.9,16.7$, and 19.4 in eyes on one, two, and three glaucoma medications, respectively [9]. Alongside such studies correlating medications with ocular surface disease, the medication reductions consistently observed after stent implantation also are well known and widely documented [16-31]. Thus, it stands to reason that stent implantation would result in ocular surface improvement. However, to our knowledge, the present study contributes some of the first data on changes in ocular surface disease measurements before and after stent implantation.

Besides ocular surface benefits, reducing medication burden also can positively impact patient adherence. Suboptimal medication adherence is widely acknowledged to be a key limitation of topical glaucoma treatments, with an estimated $50 \%$ of patients not using medications as prescribed [38]. This is particularly relevant for the approximately $40-50 \%$ of glaucoma patients who are on multiple medications, as adherence is known to drop dramatically when more than 1 medication is prescribed [10, 11]. Reducing exposure to medications and preservatives also can preserve 
conjunctival health, thereby reducing the risk of failure of future subconjunctival glaucoma surgery $[12,13]$.

Although the study's focus was on the impact of iStent or iStent inject surgery on ocular surface parameters and medication burden, the significant IOP reductions achieved also deserve mention. Even as medications were reduced 2.5 -fold, mean IOP was reduced by nearly $3 \mathrm{mmHg}$. This reduction is especially noteworthy given the cohort's relatively low preoperative mean IOP $(17.4 \mathrm{mmHg})$ and the well-known observation of smaller IOP reductions with lower preoperative IOP [17].

This study is not without limitations. There was no control group of cataract surgery alone or fellow-eye control. As is frequently done in single-arm clinical studies, patients' preoperative measurements served as their own control, although we acknowledge that this does not replace a true control group. The sample size was modest, as this was a pilot study, so future studies may include greater numbers of participants or sites. Another possible direction for future research could be to examine whether intracameral or preservative-free postoperative medications could have a different effect compared to topical medications containing preservatives. As is common in OSD studies, there was inconsistent correlation between patients' symptoms (i.e., OSDI score) and objective clinical tests (i.e., Efron, Oxford, FTBUT). However, given that the tests were completed by the same clinicians in the same patients over time, the preoperative versus postoperative comparison is still meaningful. The study was unmasked, leaving open the possibility of bias in clinicians' observations and patients' perception of their own symptoms. Study follow-up extended up to 3 months, and longer-term data may be needed to detect chronic changes in corneal and conjunctival health after stent surgery.

Limitations notwithstanding, this study contributes useful evidence on a topic currently lacking in the MIGS literature. Ocular surface health plays a central role in patient adherence, quality of life, and outcomes of future conjunctival surgery in patients with glaucoma. Implantation of iStent or iStent inject with cataract surgery produced significant improvements in ocular surface health, alongside significant reductions in IOP and medications. Thus, ocular surface improvement may be an additional postoperative benefit for doctors and patients to consider as they are evaluating glaucoma treatment options.

\section{CONCLUSIONS}

This prospective interventional study evaluated changes in patients' ocular surface symptoms and clinical signs through 3 months following iStent or iStent inject implantation with cataract surgery. Outcomes showed significant improvement in OSD parameters, as well as significant IOP and med reductions, with excellent safety. These findings constitute some of the first data on OSD changes after MIGS surgery. Given the high prevalence of OSD in glaucoma patients, and the increasing utilization of MIGS worldwide, such data are particularly germane to the ophthalmology community today.

\section{ACKNOWLEDGEMENTS}

We thank the participants in this study.

Funding. The investigators received an investigator-initiated study (IIS) grant, editorial assistance (Dana Hornbeak, MD, MPH of Glaukos Corporation), and Rapid Service Fees from Glaukos Corp. (San Clemente, CA, USA).

Authorship. All named authors meet the International Committee of Medical Journal Editors (ICMJE) criteria for authorship for this article, take responsibility for the integrity of the work as a whole, and have given their approval for this version to be published.

Disclosures. Justin Schweitzer: Bausch and Lomb: Speaker/Consultant, Sight Sciences: Consultant, Alcon: Speaker/Consultant, Sun Pharma: Consultant, Allergan: Speaker/Consultant, Equinox: Investor,Reichert: Consultant, Aerie: Speaker/Consultant, Ocular Therapeutix: 
Consultant, EyePoint Pharmaceuticals: Consultant, Johnson \& Johnson: Speaker/Consultant, Horizon Pharma: Consultant,Glaukos: Speaker/ Consultant, Quidel: Consultant. Whitney Hauser: TearLab: Advisory Board member, Paragon BioTeck: Advisory Board member, Johnson \& Johnson Vision: Speaker/Consultant, Novartis: Speaker/Consultant, Lumenis: Consultant/ Speaker, CoolDoctors: Consultant, Allergan: Speaker/Consultant, Alcon: Constant/Speaker, Bruder Medical: Consultant, BioTissue: Speaker/ Consultant, Sight Sciences, Inc.: Consultant, Eyevance Pharmaceuticals: Consultant, Kala Pharmaceuticals: Consultant, Glaukos: Consultant/Research, Sun Pharmaceuticals: Consultant, BlephEx: Consultant, Science Based Health: Consultant, Bausch \& Lomb: Consultant, Triad Life Science: Consultant, Horizon Therapeutics: Consultant, Sparca Corporation: Consultant, Osmotica Pharmaceuticals: Consultant, Keeler: Consultant, Oyster Point: Consultant, Dompe Pharmaceuticals: Consultant, Ocular Therapeutix: Consultant, Horizon Pharmaceuticals: Consultant, Keeler: Consultant, Signal Ophthalmic Consulting: Founder/Senior Consultant, DryEyeCoach.com: Founder/CEO, Ocular Surface Society of Optometry (OSSO): Board member, Optometric Cornea Cataract and Refractive Surgery (OCCRS): Board member, Primary Care Optometry News: Editorial Board member, Optometry Times: Editorial Board member, Optometric Management: Columnist. Mitch Ibach: Aerie Pharmaceuticals: Consultant/Lecturer, Alcon: Lecturer, Allergan: Consultant, Equinox: Investor, Glaukos: Consultant/Lecturer, Ocular Therapeutix: Consultant, SightSciences: Consultant. Brandon Baartman: Eyegate Pharmaceuticals: Investor, Equinox: Consultant, Glaukos: Lecturer, Investor, SightSciences: Consultant, Lecturer. John Berdahl: Alcon: Consultant, Allergan: Consultant, Avedro: Consultant/Lecturer, Aurea Medical: Consultant, Bausch and Lomb: Consultant, CorneaGen: Consultant/Lecturer, Dakota Lions Eye Bank: Advisory Board Member, Equinox: Investor, Expert Opinion: Investor. Glaukos: Consultant/Lecturer, Gore: Consultant, Imprimis: Consultant/Investor, iRenix: Consultant, Johnson \& Johnson: Consultant, Kala: Consultant, Kedalion: Consultant. MELT
Pharmaceuticals: Consultant, MicroOptx: Consultant, New World Medical Consultant/Lecturer, Ocular Surgical Data: Consultant, Ocular Theraputix: Consultant. Omage Ophthalmic: Consultant, Orasis: Consultant, Oyster Point: Consultant. RxSight: Consultant, Surface Inc: Consultant, Tarsus: Consultant, Tear Clear: Consultant, ViaLase: Consultant, Vittamed: Consultant, Verana Health: Consultant, Visionary Ventures: Consultant/Investor, Zeiss: Consultant. Subba Gollamudi, Andrew Crothers and John Linn have nothing to declare.

Compliance with Ethics Guidelines. Ethics approval was obtained from the Institutional Review Board (IRB) of the Southern College of Optometry (Memphis, Tennessee). All procedures performed were in accordance with the 1964 Declaration of Helsinki and its later amendments or comparable ethical standards. Informed consent was obtained from all participants.

Data Availability. The datasets generated during and/or analyzed during the current study are available from the corresponding author on reasonable request.

Open Access. This article is licensed under a Creative Commons Attribution-NonCommercial 4.0 International License, which permits any non-commercial use, sharing, adaptation, distribution and reproduction in any medium or format, as long as you give appropriate credit to the original author(s) and the source, provide a link to the Creative Commons licence, and indicate if changes were made. The images or other third party material in this article are included in the article's Creative Commons licence, unless indicated otherwise in a credit line to the material. If material is not included in the article's Creative Commons licence and your intended use is not permitted by statutory regulation or exceeds the permitted use, you will need to obtain permission directly from the copyright holder. To view a copy of this licence, visit http://creativecommons.org/licenses/by$\mathrm{nc} / 4.0 /$. 


\section{REFERENCES}

1. Skalicky SE, Goldberg I, McCluskey P. Ocular surface disease and quality of life in patients with glaucoma. Am J Ophthalmol. 2012;153(1-9):e2.

2. Stapleton F, Alves M, Bunya VY, et al. TFOS DEWS II epidemiology report. Ocul Surf. 2017;15:334-65.

3. Moss SE, Klein R, Klein BE. Prevalence of and risk factors for dry eye syndrome. Arch Ophthalmol. 2000;118:1264-8.

4. Leung EW, Medeiros FA, Weinreb RN. Prevalence of ocular surface disease in glaucoma patients. J Glaucoma. 2008;17(5):350-5.

5. Mylla Boso AL, Gasperi E, Fernandes L, et al. Impact of ocular surface disease treatment in patients with glaucoma. Clin Ophthalmol. 2020;14:103-11.

6. Baudouin C, Garcher C, Haouat N, et al. Expression of inflammatory membrane markers by conjunctival cells in chronically treated patients with glaucoma. Ophthalmology. 1994;101:454-60.

7. Baudouin C, Liang H, Hamard P, et al. The ocular surface of glaucoma patients treated over the long term expresses inflammatory markers related to both T-helper 1 and T-helper 2 pathways. Ophthalmology. 2008;115:109-15.

8. Tirpack AR, Vanner E, Parrish J, et al. Dry eye symptoms and ocular pain in veterans with glaucoma. J Clin Med. 2019;8:1076. https://doi.org/10. 3390/jcm8071076.

9. Fechtner RD, Godfrey DG, Budenz D, et al. Prevalence of ocular surface complaints in patients with glaucoma using topical intraocular pressure-lowering medications. Cornea. 2010;29:618-21.

10. Robin AL, Covert D. Does adjunctive glaucoma therapy affect adherence to the initial primary therapy? Ophthalmology. 2005;112:863-8.

11. Siani SD, Schoenfeld P, Kaulback K, et al. Effect of dosing frequency on adherence in chronic diseases. Am J Manag Care. 2009;15(6):22e33.

12. Broadway D, Hitchings R, Grierson I. Topical antiglaucomatous therapy: Adverse effects on the conjunctiva and implications for filtration surgery. J Glaucoma. 1995;4:136.

13. Johnson DH, Yoshikawa $\mathrm{K}$, Brubaker RF, et al. The effect of long-term medical therapy on the outcome of filtration surgery. Am J Ophthalmol. 1994;117: 139-48.
14. Nordmann JP, Auzanneau N, Ricard S, et al. Vision related quality of life and topical glaucoma treatment side effects. Health Qual Life Outcomes. 2003; 1:75.

15. Rossi GC, Tinelli C, Pasinetti GM, et al. Dry eye syndrome-related quality of life in glaucoma patients. Eur J Ophthalmol. 2009;19:572-9.

16. Samuelson TW, Katz LJ, Wells JM, et al. Randomized evaluation of the trabecular micro-bypass stent with phacoemulsification in patients with glaucoma and cataract. Ophthalmology. 2011;118: 459-67.

17. Ferguson TJ, Berdahl JP, Schweitzer JA, et al. Clinical evaluation of a trabecular micro-bypass stent with phacoemulsification in patients with openangle glaucoma and cataract. Clin Ophthalmol. 2016;10:1767-73.

18. Neuhann TH, Hornbeak DM, Neuhann RT, et al. Long-term effectiveness and safety of trabecular micro-bypass stent implantation with cataract surgery in patients with glaucoma or ocular hypertension: 5-year outcomes. J Cataract Refract Surg. 2019;45(3):312-20.

19. Ferguson TJ, Swan R, Ibach M, et al. Trabecular microbypass stent implantation with cataract extraction in pseudoexfoliation glaucoma. J Cataract Refract Surg. 2017;43(5):622-6.

20. Ferguson T, Swan R, Ibach M, et al. Evaluation of a trabecular microbypass stent with cataract extraction in severe primary open-angle glaucoma. J Glaucoma. 2018;27(1):71-6.

21. Ferguson TJ, Ibach M, Schweitzer J, et al. Trabecular microbypass stent implantation with cataract extraction in pigmentary glaucoma. Clin Exp Ophthalmol. 2019. https://doi.org/10.1111/ceo. 13638.

22. Gallardo MJ, Supnet RA. Three-year outcomes of combined trabecular micro-bypass and phacoemulsification in a predominantly Hispanic population with primary open-angle glaucoma. Clin Ophthalmol. 2019;13:869-79.

23. Fechtner RD, Voskanyan L, Vold SD, et al. Five-year, prospective, randomized, multi-surgeon trial of two trabecular bypass stents versus prostaglandin for newly-diagnosed open-angle glaucoma. Ophthalmology Glaucoma. 2019;2(3):156-66.

24. Samuelson TW, Sarkisian SR Jr, Lubeck DM, for the iStent inject Study Group, et al. Prospective, randomized, controlled pivotal trial of istent inject trabecular micro-bypass in primary open-angle glaucoma and cataract: two-year results. Ophthalmology. 2019;126(6):811-21. 
25. Manning D. Real-world case series of iStent or iStent inject trabecular micro-bypass stents combined with cataract surgery. Ophthalmol Ther. 2019;8(4): 549-61.

26. Neuhann R, Neuhann T. Second-generation trabecular micro-bypass stent implantation: Retrospective analysis after 12- and 24-month follow-up. Eye Vis (Lond). 2020;10(7):1.

27. Hengerer FH, Auffarth GU, Riffel C, et al. Prospective, non-randomized, 36-month study of secondgeneration trabecular micro-bypass stents with phacoemulsification in various types of glaucoma. Ophthalmol Ther. 2018;7(2):405-15.

28. Hengerer FH, Auffarth GU, Riffel C, et al. Secondgeneration trabecular micro-bypass stents as standalone treatment for glaucoma: a 36-month prospective study. Adv Ther. 2019;36(7):1606-17.

29. Salimi A, Lapointe J, Harasymowycz P. One-year outcomes of second-generation trabecular microbypass stents (istent inject) implantation with cataract surgery in different glaucoma subtypes and severities. Ophthalmol Ther. 2019;8(4):563-75.

30. Clement CI, Howes F, Ioannidis AS, et al. One-year outcomes following implantation of second-generation trabecular micro-bypass stents in conjunction with cataract surgery for various types of glaucoma or ocular hypertension: multicenter, multi-surgeon study. Clin Ophthalmol. 2019;13:491-9.

31. Guedes RAP, Gravina DM, Lake JC, et al. One-year comparative evaluation of istent or istent inject implantation combined with cataract surgery in a single center. Adv Ther. 2019;36(10):2797-810.

32. Schiffman RM, Christianson MD, Jacobsen G, et al. Reliability and validity of the Ocular Surface Disease Index. Arch Ophthalmol. 2000;118(5):615-21.

33. Bron AJ, Evans VE, Smith JA. Grading of corneal and conjunctival staining in the context of other dry eye tests. Cornea. 2003;22:640-50.

34. Efron N, Morgan PB, Katsara SS. Validation of grading scales for contact lens complications. Ophthalmic Physiol Opt. 2001;21:17-29.

35. Wolffsohn JS, Arita R, Chalmers R, et al. TFOS DEWS II diagnostic methodology report. Ocul Surf. 2017;15:539-74.

36. Agnifili L, Brescia L, Scatena B, et al. Tear Meniscus Imaging by Anterior Segment-optical Coherence Tomography in Medically Controlled Glaucoma. J Glaucoma. 2020. https://doi.org/10.1097/IJG. 0000000000001469

37. Begley C, Chalmers RL, Abetz L, et al. The relationship between habitual patient-reported symptoms and clinical signs among patients with dry eye of varying severity. Invest Ophthalmol Vis Sci. 4753e;44:4753e61.

38. Brown MT, Bussell JK. Medication adherence: WHO cares? Mayo Clin Proc. 2011;86(4):304-14. 